\title{
Myosin Vc, a Member of the Actin Motor Family Associated with Rab8, Is Involved in the Release of DV2 from HepG2 Cells
}

\author{
Xiao-Feng $\mathrm{Xu}^{\mathrm{a}}$ Zong-Tao Chen ${ }^{\mathrm{b}} \mathrm{Na} \mathrm{Gao}^{c}$ Jun-Lei Zhang ${ }^{\mathrm{b}}$ Jing $\mathrm{An}^{\mathrm{d}}$ \\ Departments of a Medical Genetics, ${ }^{\mathrm{b}}$ Microbiology, and ${ }^{\mathrm{c} H i s t o l o g y}$ and Embryology, Third Military Medical \\ University, Chongqing, and d Department of Microbiology, School of Basic Medical Sciences, Capital Medical \\ University, Beijing, PR China
}

\section{Key Words}

Dengue virus · HepG2 cells $\cdot$ Rab8 $\cdot$ Myosin Vc

\begin{abstract}
Objective: The pathogenesis of the dengue virus (DV) infection has not been well defined. We have reported that actin and Rab8 are involved in DV2 infection. Myosin Vc (Myo5c) is a novel member of the class $V$ myosins and regulates the actin-mediated membrane trafficking associated with Rab8. In this study, the involvement of Myo5c in the release of DV2 was investigated in HpeG2 cells. Methods: Distributions of actin, Myo5c, DV2 and Rab8 were revealed by fluorescent staining. HepG $2^{\text {Myo5c-tail }}$ cells expressing a dominant-negative mutant of Myo5c were constructed by transfection and were assessed by Western blotting. The viral titers were detected by plaque assay, and the expression of Rab8 was analyzed by flow cytometry. Results: DV2 infection altered the distribution pattern of Myo5c, which might be associated with the depolymerization of actin, though colocalization rates of Myo5c with DV2 or actin were low. Furthermore, the release of DV2, but not the intracellular viral production, was reduced from HepG2 ${ }^{\text {Myo5c-tail }}$ cells. Moreover, Myo5c colocalized with Rab8 and an increase of Rab8 was associated with the decrease of the viral release caused by the Myo5c tail. Conclusions: Our data suggest that Myo5c associated with Rab8 is involved in the release of DV2 from HepG2 cells.
\end{abstract}

\section{KARGER}

Fax +4161306 1234 E-Mail karger@karger.ch www.karger.com (c) 2009 S. Karger AG, Basel

0300-5526/09/0525-0258\$26.00/0

Accessible online at:

www.karger.com/int

\section{Introduction}

Dengue virus (DV) is an enveloped, positive singlestranded RNA virus belonging to the family Flaviviridae [1]. The viruses, including 4 distinct serotypes (DV1DV4), are transmitted to humans by mosquitos [1]. These viruses cause a wide range of symptoms from an unapparent or mild disease (dengue fever) to severe, lifethreatening complications (dengue hemorrhagic fever/ dengue shock syndrome). Recently, its infection has reemerged as a more and more severe threat for human health, especially in the tropical and subtropical regions worldwide. However, the pathogenesis of dengue fever and dengue hemorrhagic fever/dengue shock syndrome has not been characterized to date.

As obligate intracellular parasites, many viruses effectively and efficiently exploit the cytoskeleton, traffic motors and other proteins to facilitate their intracellular trafficking. Therefore, identification of the host proteins and trafficking pathways that permit viruses to fulfill their infection cycles in cells will provide new insight into the molecular mechanism of DV2 infection and may allow for the development of novel antiviral therapeutics.

In previous studies, we found that both actin and Rab8, a regulator of vesicular traffic from the Golgi apparatus to the plasma membrane, were involved in DV2

X.-F.X. and Z.-T.C. have equally contributed to this work.
Jing An

Department of Microbiology, School of Basic Medical Sciences

Capital Medical University

Beijing 100069 (PR China)

Tel./Fax +86 108391 1741, E-Mail anjing60@yahoo.com.cn 
infection [2, 3]. As actin-mediated movements of Rab8positive vesicles are largely dependent on Rab8 activity, Rab8 is ideal in regulating specific recruitment of motor proteins to defined vesicles [4]. As a novel member of the class $\mathrm{V}$ myosins, which is the most efficient and processive class in actin-mediated transport, myosin Vc (Myo5c) selectively colocalizes with membrane compartments containing Rab8 in transports of transferrin and may drive the actin-mediated membrane trafficking pathway [5]. Thus, we wanted to investigate whether Myo5c, which is a motor of actin and interacts with Rab8, is also involved in the process of DV2 infection.

Although the liver is not a major target organ, the susceptibility of the liver and liver-derived cells in DV infection has been revealed [6-9]. Therefore, HepG2, a human hepatocarcinoma cell line, was used in this study to investigate the role of Myo5c on DV2 infection. Our results suggest that Myo5c may be involved in the release of DV2 and this involvement has a strong relationship with Rab8.

\section{Materials and Methods}

\section{Cell Lines and Virus}

A HepG2 (ATCC) cell line was cultured in Dulbecco's modified Eagle's minimum essential medium (DMEM, Gibco) with $10 \%$ fetal bovine serum (FBS). Vero cells were cultured in minimal essential medium (Gibco) with 5\% FBS and used for plaque assay, and an Aedes albopictus cell line (C6/36) was grown in RPMI 1640 (Gibco) with 10\% FBS.

DV2 (strain $\operatorname{Tr} 1751$ ) was isolated from a patient with dengue fever, propagated in C6/36 cells and stored at $-70^{\circ}$. Viral titers were detected by plaque assay using a Vero cell monolayer culture under $1 \%$ methylcellulose overlay medium.

\section{Antibodies}

The anti-Rab8 monoclonal antibody was from BD Biosciences. Fluorescein isothiocyanate (FITC)-conjugated goat antimouse IgG1 and Cy3-labelled goat anti-rabbit IgG were from Sigma and Chemicon, respectively.

Rabbit anti-DV2 polyclonal antibody (pAb) had been prepared in our lab and used in our previous work [3]. To obtain rabbit anti$\mathrm{Rab} 8 \mathrm{pAb}$, a protein containing $6 \times \mathrm{His}$ in the $\mathrm{N}$-terminal followed by 122 amino acids of Rab8 in the C-terminal was expressed in Escherichia coli JM109. After purification with a Ni affinity column, the truncated Rab8 protein $(15 \mathrm{kDa})$ was used to immunize adult rabbits with the standard immune procedure, after which the antiserum was obtained. To produce mouse anti-Myo5c pAb, a protein containing 297 amino acids of Myo $5 \mathrm{c}$ in $\alpha$-helical coiledcoil tail domain was prepared by a similar method as described above. The truncated Myo5c protein, a $42-\mathrm{kDa}$ fusion protein containing $6 \times$ His in the $\mathrm{N}$-terminal, was used to immunize 8 week-old Balb/c mouse to obtain the antiserum. The specificity of the antibodies was measured by Western blotting and indirect immunofluorescence staining.

Myosin Vc Is Involved in the Release of DV2
Plasmids and Transfections

To construct a dominant-negative mutant of Myo5c, the DNA fragment encoding the entire tail of Myo5c (aa 902-1742) was amplified by RT-PCR from the total RNA of human gastric mucosa, digested with $\mathrm{XbaI} / \mathrm{XhoI}$ and then directly cloned into a pCI-neo vector (Promega).

To express the Myo5c tail, HepG2 cells were transfected with the constructed plasmid (pCI-Myo5c-tail) and a control plasmid (pCI-neo) using Lipofectamine 2000 (Invitrogen) following the manufacturer's instructions. The cells were cultured with DMEM containing $10 \% \mathrm{FBS}$ and $600 \mu \mathrm{g} / \mathrm{ml}$ of the neomycin analogue G418 sulfate (Geneticin). After selection for 14 days, single cell clones stably transfected with the above plasmids were obtained and named HepG2 ${ }^{\mathrm{Myo5c} \text {-tail }}$ and HepG2 $2^{\mathrm{pCI}}$ cells, respectively. Western blotting evaluated how well established they were.

\section{Designs of DV2 Infection Experiments}

To detect infectious DV2 produced in or released from cells at time points of 8,24 and 48 h p.i., HepG $2^{\text {Myo5c-tail }}$ and HepG2 $2^{\text {pCI }}$ cells were infected with DV2 at a MOI of 10 for $1 \mathrm{~h}$ at $37^{\circ}$. After excess or unbound viruses were removed by extensive washing, cells were maintained in DMEM with $2 \% \mathrm{FBS}$ at $37^{\circ}$ until the time points. Plaque formations of cell fractions or supernatant viruses were detected and 3 independent experiments were performed for each time point.

Flow Cytometric Analysis

Expression of Rab8 in HepG2 $2^{\text {Myo5c-tail }}$ and HepG2 ${ }^{\mathrm{PCI}}$ cells was determined by flow cytometry. Briefly, cells were trypsinized, washed in phosphate buffered saline (PBS), fixed in $4 \%$ paraformaldehyde in PBS and permeabilized with permeabilization solution (Jingmei). They were blocked with $1 \%$ BSA and then incubated with the anti-Rab8 monoclonal antibody on ice for $30 \mathrm{~min}$. After being washed, cells were incubated with a FITC-conjugated goat anti-mouse IgG1 on ice for $20 \mathrm{~min}$. Then cells were analyzed on a FACScan cytofluorometer (Becton Dickinson). A total of $10^{4}$ cells were analyzed for each sample. The fluorescence produced by HepG $2^{\mathrm{PCI}}$ cells with or without primary antibody was used as the control and background, respectively. Dead cells stained with propidium iodide were excluded from the analysis.

\section{Western Blotting}

HepG2 ${ }^{\mathrm{pCI}}$ and HepG2 $2^{\mathrm{Myo5c} \text {-tail }}$ cells were lysed in $1 \times$ Laemmli loading buffer. After incubation at $100^{\circ}$ for $5 \mathrm{~min}$, samples were subjected to a $10 \%$ SDS polyacrylamide gel and electrophoretically transferred onto nitrocellulose membranes (Dingguo). After incubation with $5 \%$ nonfat milk in Tris-Tween buffered saline (TTBS, $\mathrm{pH} 8.0,10 \mathrm{~mm}$ Tris, $150 \mathrm{~mm} \mathrm{NaCl}$ and $0.05 \%$ Tween 20) for $1 \mathrm{~h}$, the membranes were incubated with mouse anti-Myo5c pAb overnight at $4^{\circ}$. The membranes were then washed 3 times in TTBS buffer and incubated with horseradish peroxidase-conjugated goat anti-mouse IgG (Zhongshan Golden Bridge) at $37^{\circ}$ for $1 \mathrm{~h}$. Blots were washed 3 times with TTBS and developed with 3,3'-Diaminobenzidine (DAB, Sigma). Images were processed using Adobe Photoshop (Adobe Systems).

Indirect Immunofluorescence Staining

Cells were grown on glass coverslips and infected with DV2 or heat-inactivated DV2 for $1 \mathrm{~h}$ at $37^{\circ}(\mathrm{MOI}=10)$. At $24 \mathrm{~h}$ p.i., cells were subjected to immunofluorescence staining. In brief, cells 


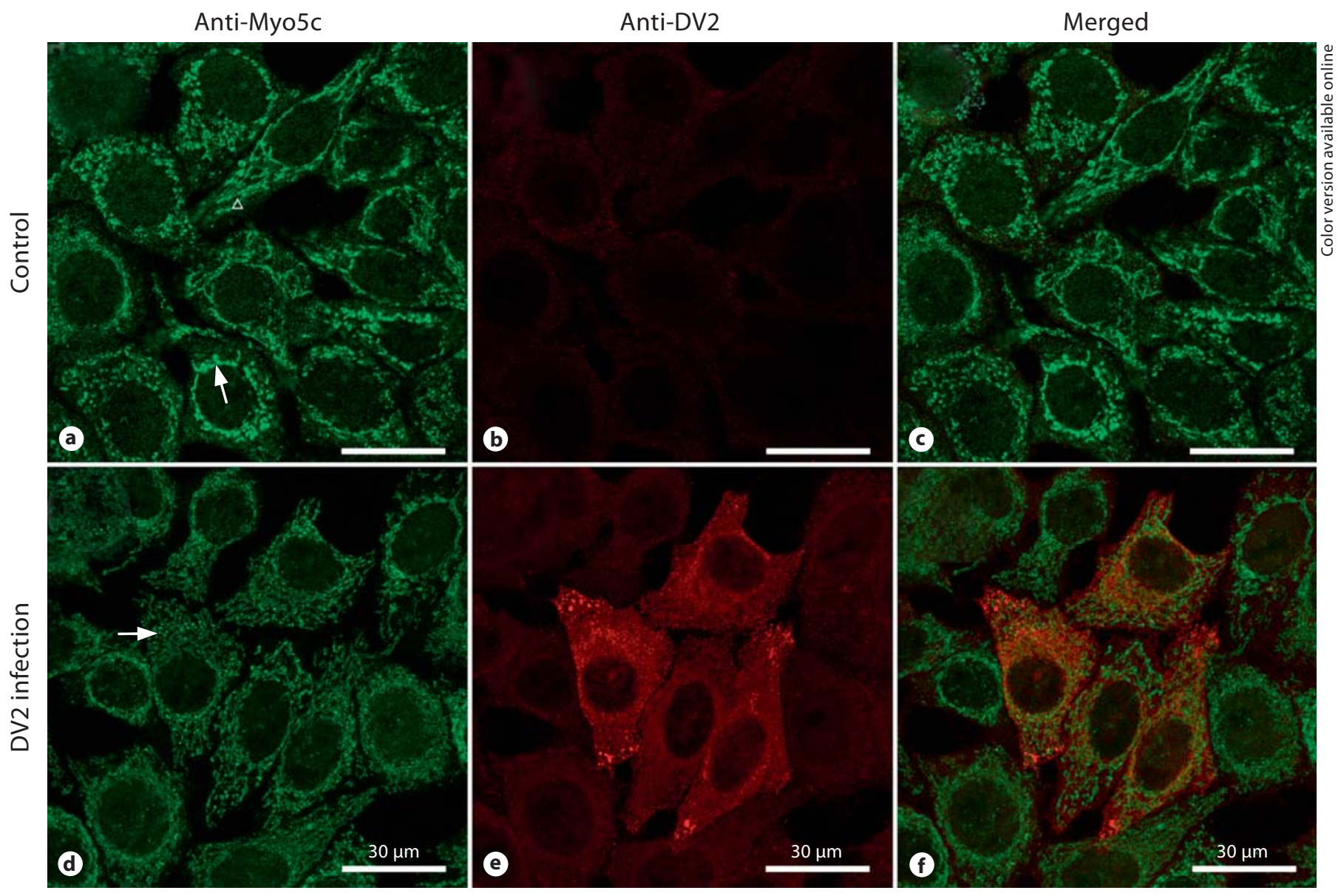

Fig. 1. Distribution of Myo5c and DV2 antigens in HepG2 cells at 24 h p.i. HepG2 cells infected by heat-inactivated DV2 or DV2 were double-stained to detect Myo5c (a, d) and DV2 (b, e). Myo5c clearly located around the nucleus as aggregated patches (arrow) or in the cytoplasm like flexible filaments (triangle, a). DV2 infec- tion induced the disintegration of Myo5c (arrow, d), but the colocalization of Myo5c with DV2 antigens was rarely observed in the merged images $(\mathbf{c}, \mathbf{f})$. Cells infected by heat-inactivated DV2 were used as the control. Bar $=30 \mu \mathrm{m}$. were fixed with $4 \%$ polyformaldehyde in PBS and then permeabilized with PBS containing $0.2 \%$ Triton X-100. After being washed and blocked with PBS containing $1 \%$ BSA, cells were incubated with rabbit anti-DV2 pAb, rabbit anti-Rab8 pAb or mouse antiMyo5 $\mathrm{c}$ pAb at $4^{\circ}$ overnight and were followed by Cy3-labelled goat anti-rabbit IgG or FITC-conjugated goat anti-mouse IgG1 and/or phalloidin-TRITC (Sigma), respectively. The specimens were observed with a confocal laser microscope (Leica TCS SP5). Images were processed by Adobe Photoshop and the colocalization rate was analyzed by Leica's software system.

\section{Statistical Analysis}

Data were analyzed using the SPSS software (Version 12.0 for Windows, SPSS Inc.) by Student's t test. Differences were deemed statistically significant when $\mathrm{p}<0.05$.

\section{Results}

\section{The Distribution of Myo5c Is Disrupted by DV2 \\ Infection}

To localize the Myo5c and DV2 in cells, the HepG2 cells infected by DV2 or inactive virions were subjected to double-staining with anti-Myo5c and anti-DV2 pAbs (fig. 1). In most heat-inactivated DV2-infected cells, Myo5c clearly located in the perinuclear region as aggregated patches or in the cytoplasm like flexible filaments (fig. 1a), while its fluorescence became faint and was altered to small puncta distributed in the cytoplasm in infected cells at $24 \mathrm{~h}$ p.i. (fig. 1d). The location of the DV2 antigen was in the cytoplasm, which is similar to our previous observation (fig. 1e) [3]. Although DV2 infection induced this disintegration, 


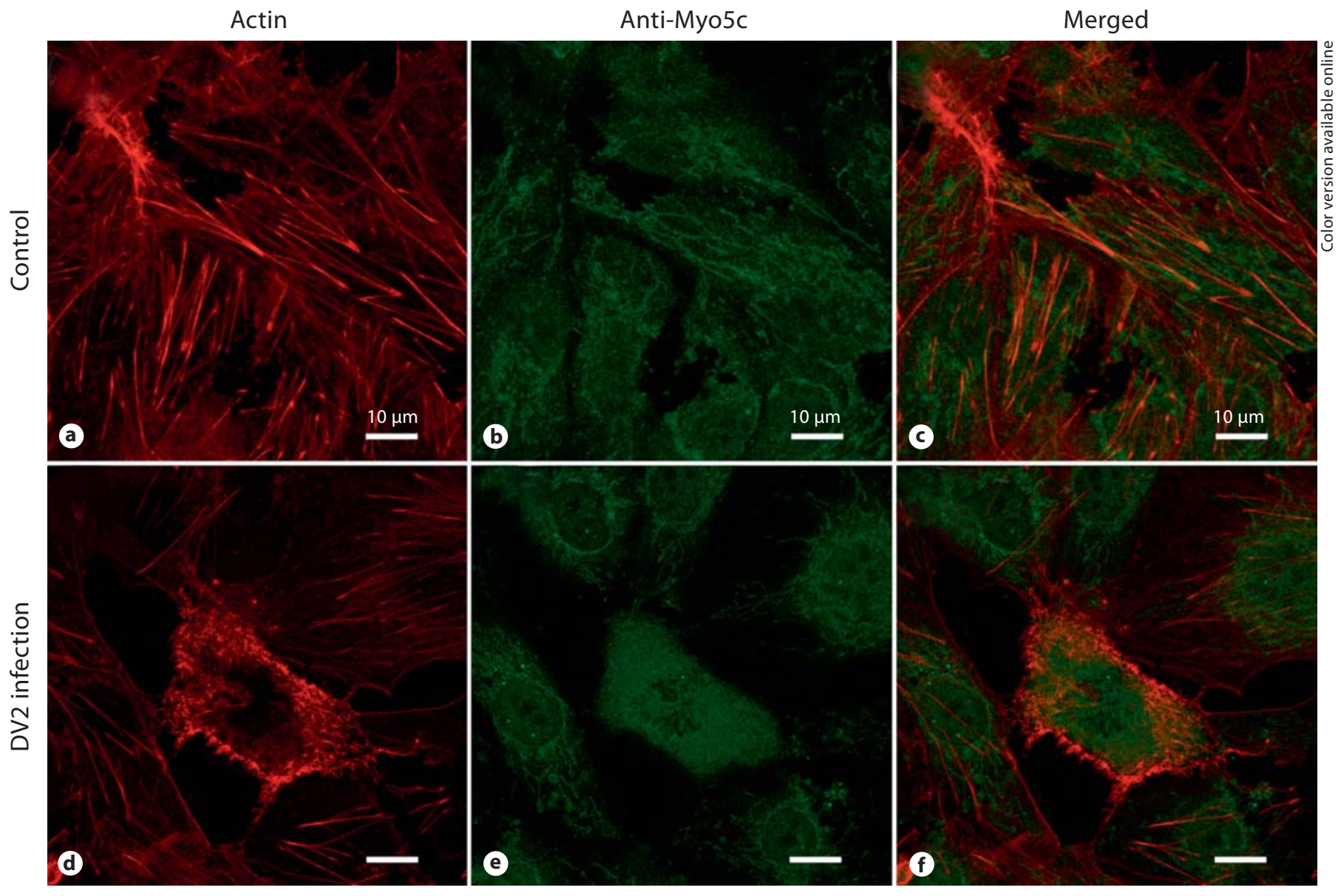

Fig. 2. Distribution of actin and Myo5c in HepG2 cells. HepG2 cells infected by heat-inactivated DV2 or DV2 were labelled with phalloidin-TRITC (a, d) and anti-Myo5c pAb (b,e) at $24 \mathrm{~h}$ p.i. The merged images show no obvious colocalization of Myo5c with actin $(\mathbf{c}, \mathbf{f})$. Cells infected by heat-inactivated DV2 were used as the control. Bar $=10 \mu \mathrm{m}$. the colocalization of Myo5c with DV2 antigens was rarely observed (fig. 1f). The observation suggests that Myo5c may be associated with the process of DV2 infection.

As Myo5c is a motor of actin and a DV2 infection causes the depolymerization of actin $[2,5]$, we hypothesized the disintegration of Myo5c would be related to the change of actin after DV2 infection. To test this hypothesis, HepG2 cells infected by DV2 or inactive virions were labelled with phalloidin-TRITC and anti-Myo5c pAb (fig. 2). Most of heat-inactivated DV2-infected HepG2 cells showed the typical morphology of F-actin, but they were quite different from reports by Rodriguez and Cheney [5] and Marchelletta [10], who showed that actin located under the plasma membrane-like rings in sections of the colon, pancreas, lacrimal glands and lacrimal gland acinar cells. There was also no obvious location of Myo5c along with these actin filaments in HepG2 cells (fig. 2c). At $24 \mathrm{~h}$ after DV2 infection, the disintegration of Myo5c and depolymerization of actin were seen, while the colocalization of actin with Myo5c was rarely observed in these DV2-infected cells (fig. 2f). However, it is implied that the depolymerization of actin may affect the normal distribution of $\mathrm{Myo} 5 \mathrm{c}$, though the colocalization of actin with Myo5c is not obvious in HepG2 cells.

\section{The Construction of HepG2 Cells Stably Expressing}

the Myo5c Tail

To establish a HepG2 cell line stably expressing the dominant-negative mutant of Myo5c, we first constructed a plasmid-expressing Myo5c tail, i.e. the pCI-Myo5ctail. The plasmid was confirmed by double enzyme digestion and sequencing.

To validate that the cells stably expressed the Myo5c tail, the following experiments were applied. 


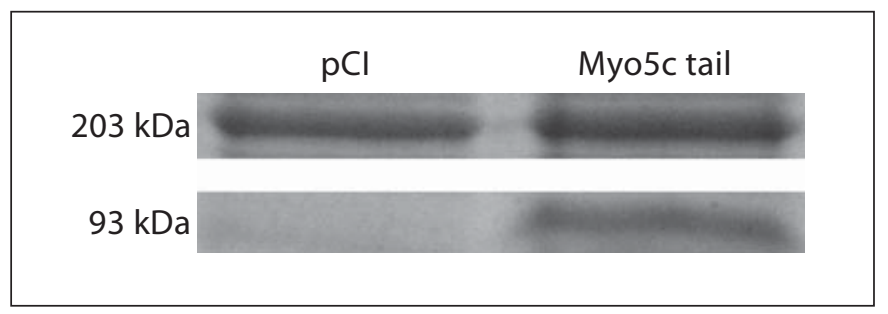

Fig. 3. Assessment of HepG2 cells stably expressing the dominantnegative mutant of Myo5c. Single-cell clones stably transfected with pCI-Myo5c-tail or pCI-neo were subjected to Western blotting. Expression of the Myo5c tail in HepG2 $2^{\text {Myo5c-tail }}$ cells was confirmed. Our mouse anti-Myo5c pAb specifically reacted with both the endogenous Myo5c protein $(203 \mathrm{kDa})$ in $\mathrm{HepG}^{\mathrm{pCl}}$ and HepG2 $2^{\text {Myo5c-tail }}$ cells, and the Myo5c tail $(93 \mathrm{kDa})$ in HepG2 $2^{\text {Myo5c-tail }}$ cells, respectively.

HepG2 $2^{\text {Myo5c-tail }}$ cells and its control, HepG2 $2^{\text {pCI }}$, were affirmed by Western blotting. The result showed that our mouse anti-Myo5c pAb specifically reacted with both the endogenous Myo5c protein $(203 \mathrm{kDa})$ in $\mathrm{HepG}^{\mathrm{pCI}}$ and HepG2 ${ }^{\text {Myo5c-tail }}$ cells and the Myo5c tail $(93 \mathrm{kDa})$ expressed in HepG2 ${ }^{\mathrm{Myo5} \text {-tail }}$ cells (fig. 3).

\section{Myo5c Modulates the Release of DV2 from HepG2 Cells}

As Myo5c regulates the actin-mediated vesicular transport of transferrin associated with Rab8 and facilitates the exocytosis in lacrimal gland acinar cells $[5,10]$, it was inferred that the dominant-negative mutant of Myo5 $\mathrm{c}$ might interrupt the viral release and cause a decrease of the supernatant viral titers. To test this interference, we analyzed the supernatant virus released from HepG2 $2^{\text {pCI }}$ and HepG2 ${ }^{\text {Myo5c-tail }}$ cells at 8,24 and 48 h p.i. by plaque assay (fig. 4a). Compared with the control, expression of the Myo5c tail reduced the titers of progeny virus from cells by $32.5,39.4$ and $47.3 \%$ at 8,24 and $48 \mathrm{~h}$ p.i., respectively $(\mathrm{p}<0.05, \mathrm{n}=3)$. The data show that the interruption of intracellular Myo5c decreases the release of the progeny virus, suggesting its involvement in the release of DV2 from the host cells.

Because of the possibility of involvement of some traffic-related proteins in viral biosynthesis and to better understand the decrease of the supernatant virus caused by the Myo5c tail, we analyzed the virus in the cell fraction of HepG2 $2^{\mathrm{pCI}}$ and HepG2 ${ }^{\mathrm{Myo} 5 \mathrm{c}-\mathrm{tail}}$ cells at 8, 24 and $48 \mathrm{~h}$ p.i. by plaque assay (fig. $4 \mathrm{~b}$ ). Expression of the Myo5c tail only reduced the viral titers by $13.9,32.1$ and $10.4 \%$ at 8 , 24 and 48 h p.i., respectively ( $p>0.05, n=3$ ). There were also no significant differences in the statistics on these
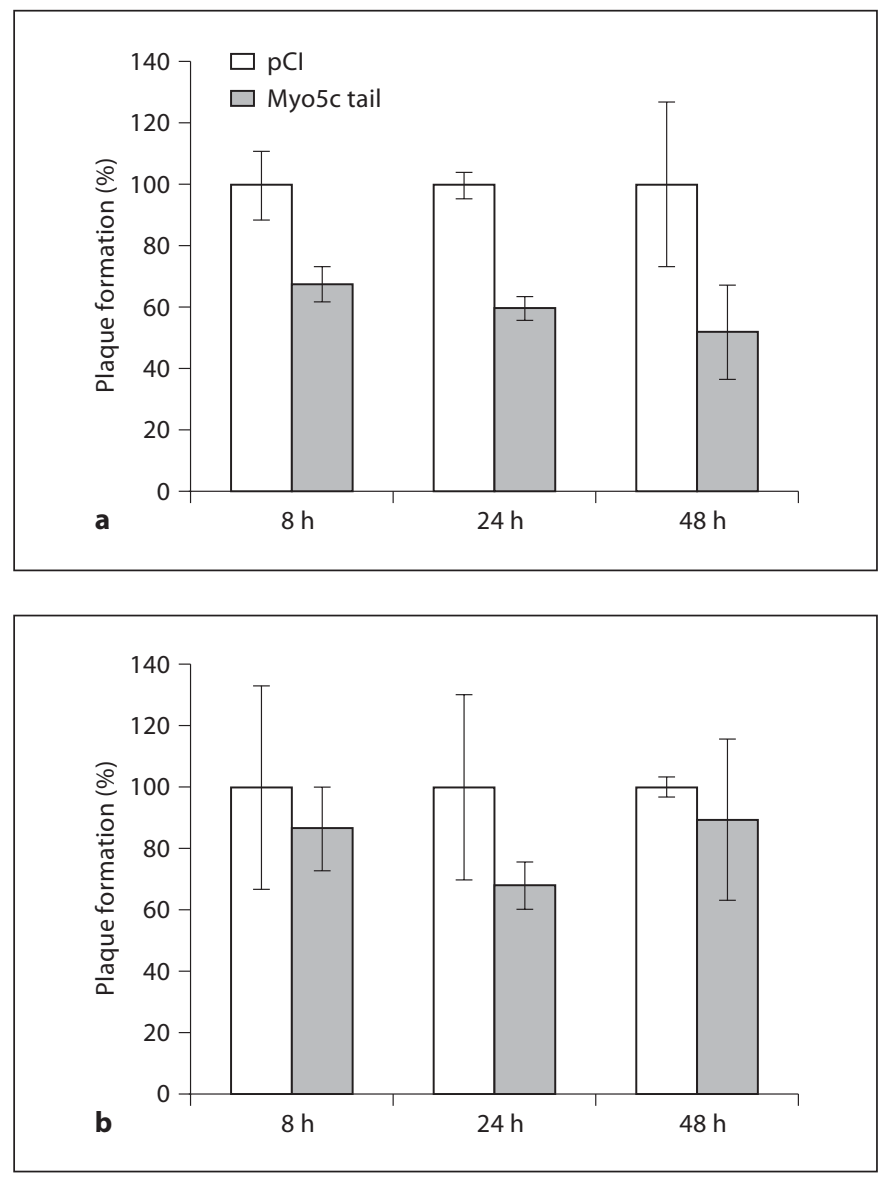

Fig. 4. The decrease in DV2 release but not the viral production caused by the Myo5c tail. The viral titers of the supernatant and

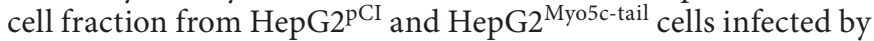
DV2 were detected by plaque assay. Expression of the Myo5c tail reduced progeny virus released to the supernatant at 8,24 and $48 \mathrm{~h}$ p.i. (a), but did not impair the intracellular production of infectious virions (b). In all experiments, $\mathrm{p}$ values $<0.05$ were considered significant $(n=3)$. Results are expressed as means $\pm S D$ from triplicates of a representative experiment.

decreases compared with the control. The data show that the interruption of intracellular Myo5c does not impair the production of DV2 in the host cells.

\section{The Decrease of Viral Release is Probably Associated with the Increase of Rab8 Expression Caused by the Myo5c Tail}

To confirm the relationship between Myo5c and Rab8, HepG2 cells were first subjected to double-staining by their pAbs. The endogenous Rab8 antigen predominantly localized, similar to previous findings, in the cytoplasm from the perinuclear region to the plasma membrane in 


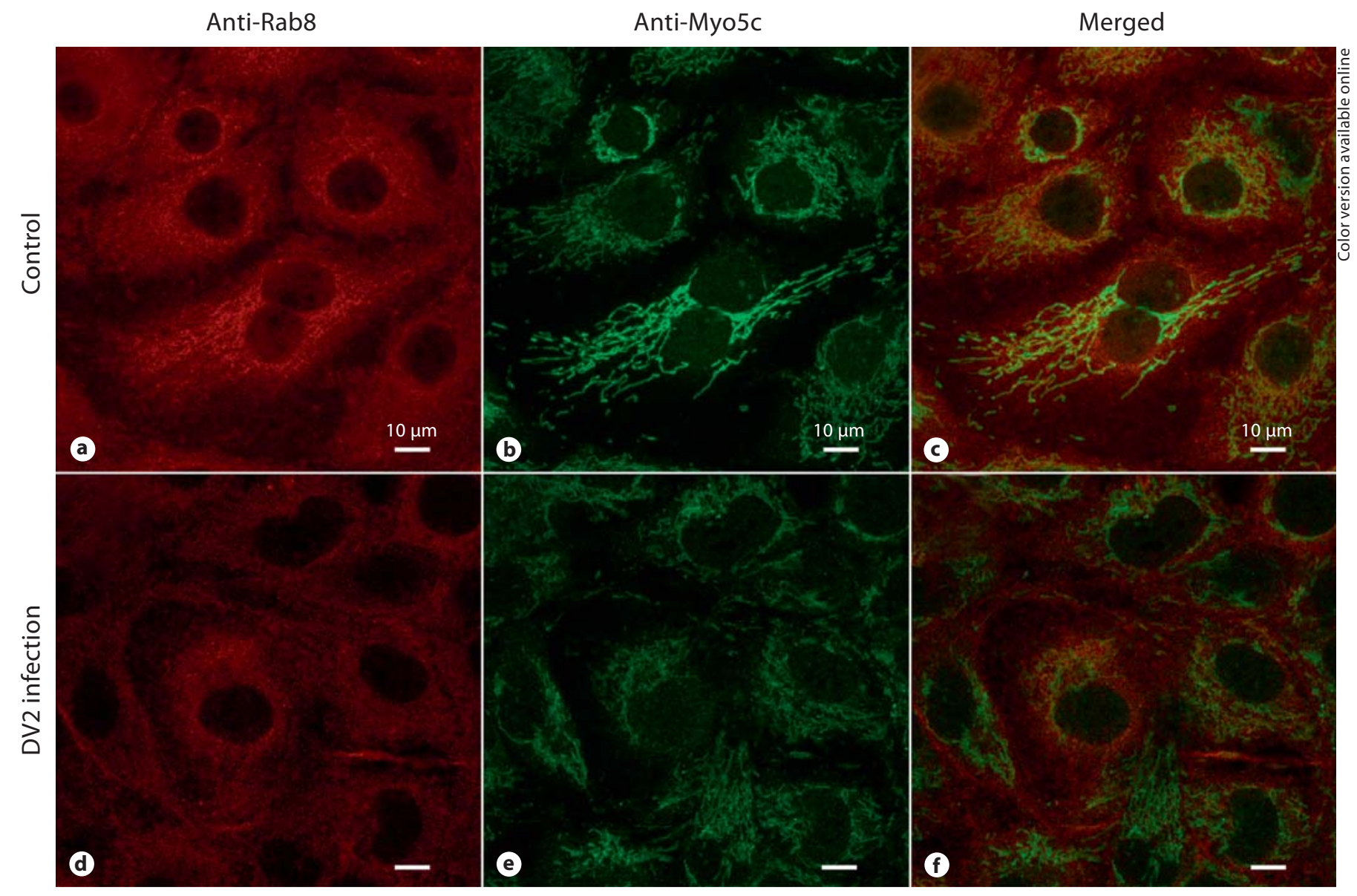

Fig. 5. The colocalization of Myo5c with Rab8 in HepG2 cells. HepG2 cells infected by heat-inactivated DV2 or DV2 were double-labelled with mouse anti-Myo5c (b, e) and rabbit anti-Rab8 (a, d) pAbs. DV2 infection induced the disintegration of Myo5c, but the colocalization of Myo5c with Rab8 was detectable in the merged images $(\mathbf{c}, \mathbf{f})$. Cells infected by heat-inactivated DV2 were used as the control. Bar $=10 \mu \mathrm{m}$.
HepG2 cells (fig. 5a) [3, 11, 12], and our mouse anti-Myo5c $\mathrm{pAb}$ specifically discriminated against Myo5c antigen in HepG2 cells (fig. 5b). By confocal analysis, Myo5c colocalized with Rab8 in the cytoplasm region where their colocalization rate was up to about $90 \%$ in some regions of interest (fig. 5c). Furthermore, there were some detectable signals of the colocalization of Myo5c with Rab8 in some regions of interest, though DV2 infection led to the disintegration of Myo5c and their colocalization became faint (fig. 5d-f). As we had found that the release of DV2 is significantly inhibited by the interruption of the functional balance of Rab8 in HepG2 cells [3], the colocalization of Myo5c with Rab8 suggests that the modulation of viral release by Myo5c is probably associated with Rab8.

Consequently, to further investigate the decrease of the viral release caused by expression of the Myo5c tail in
HepG2 cells, we detected the expression of Rab8 in HepG2 ${ }^{\mathrm{PCI}}$ and Hep G2 ${ }^{\mathrm{Myo5c} \text {-tail }}$ cells by flow cytometry (fig. 6). The fluorescence intensity of FITC represented the quantity of Rab8 antigen in cells. The result showed that Rab8 antigen significantly increased by about 1.3 -fold in HepG2 ${ }^{\text {Myo5c-tail }}$ cells as compared with HepG2 ${ }^{\text {pCI }}$ cells ( $p<$ $0.05, \mathrm{n}=3$ ). Therefore, the functional interruption of Myo5c leads to the increase of Rab8 in HepG2 cells. In previous studies, the overexpression of Rab8 could interrupt its functional state and interrupt the vesicular traffic, and we were also able to demonstrate the involvement of Rab8 in DV2 infection [3, 4, 12]. Taken together, the data suggest that one of the reasons for the decrease of the viral release from infected HepG2 ${ }^{\mathrm{Myo5c} \text {-tail }}$ cells may result from the interruption of the functional state of Rab8 in host cells. 


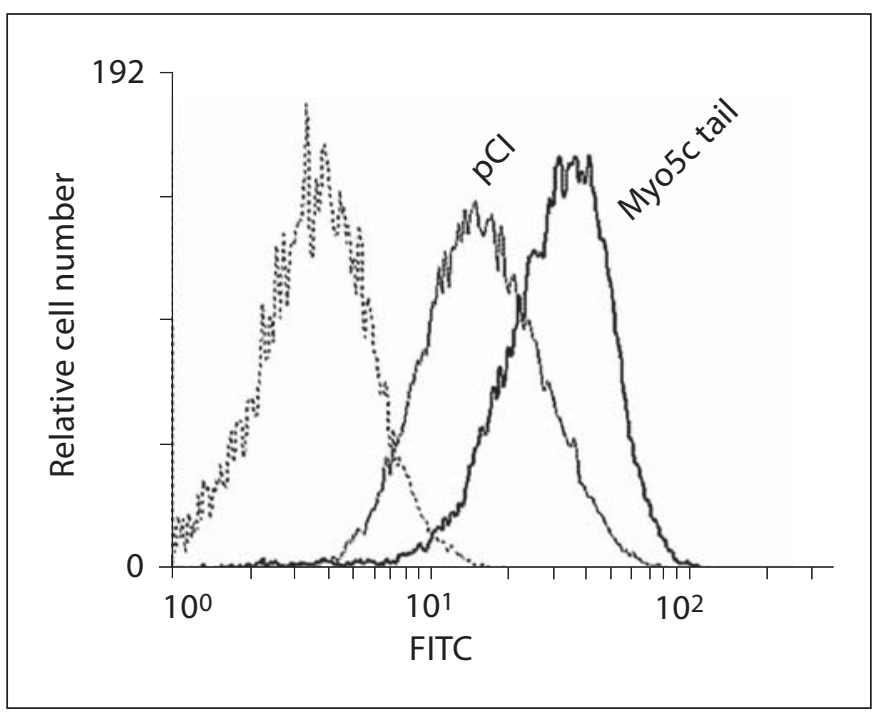

Fig. 6. An increase of Rab8 induced by the inhibition of Myo5c. The expression of Rab8 in HepG2 $2^{\mathrm{pCI}}$ and HepG2 $2^{\mathrm{Myo5c} \text {-tail }}$ cells were detected by flow cytometry. Rab8 antigen significantly increased in HepG2 ${ }^{\text {Myo5c-tail }}$ cells $(\mathrm{p}<0.05, \mathrm{n}=3)$. HepG2 ${ }^{\mathrm{pCI}}$ cells incubated with or without mouse anti-Rab8 monoclonal antibody were used as the control and background, respectively.

\section{Discussion}

According to the theory of interaction between host cells and viruses, DV is believed to utilize the living cell's exocytosis pathway to release from hosts. If this is so, then which regulator of cellular transports would be involved in the release of the progeny virions? The answer will provide more details on the life cycle of DV and maybe provide further insight into controlling dengue fever and dengue hemorrhagic fever/dengue shock syndrome and therapy.

In 2002, Rodriguez and Cheney first reported that Myo5c, a novel member of the class $V$ myosins, regulated the actin-mediated membrane trafficking of transferrin associated with Rab8 in HeLa cells [5]. In our previous works, we showed how the inhibition of the supernatant DV2 released from the host cells was induced by the interruption of either actin in ECV304 and human umbilical vesicular endothelial cells or Rab8 in HepG2 cells [2, 3]. Consequently, in this paper we were interested in finding out whether Myo5c is involved in the process of DV2 infection. We found that the distribution of Myo5c is altered by DV2 and the dominant-negative mutant of Myo5c reduces the viral release from the HepG2 cells. Therefore, our results partially support the hypothesis that DV2 utilizes the cellular traffic pathway, including the cytoskeleton, traffic motors and vesicular transports, to fulfill its life cycle in host cells.

Our previous work showed that DV2 infection leads to the obvious rearrangement of actin in ECV304 and human umbilical vesicular endothelial cells [2]. As a motor of actin [5], Myo5c may passively alter its regular localization in cells associated with the changes from fibrous actin to globular actin. This may explain why disintegration of Myo5c was found in the cytoplasm from the perinuclear region in DV2-infected HepG2 cells. Additionally, the alteration of Myo5c may interrupt the original cellular traffic and transports of newly biosynthesized virions.

Brock et al. [13] found that expression of the Myosin $\mathrm{Vb}$ (Myo5b) tail, which functions as a dominant-negative inhibitor of apical recycling endosome-mediated protein sorting in polarized Madin-Darby canine kidney cells, significantly reduces the viral yield of respiratory syncytial virus. It has been reported recently that Vpu-mediated enhancement of HIV particle release though the pericentriolar recycling endosome is also abrogated by expression of dominant-negative mutants of Rab1la and Myo5b [14]. These reports indicate that some members of the class $\mathrm{V}$ myosins are most likely to be involved in viral release according to their critical functions in cells. Interestingly, Rab8, which co-localizes with Myo5c, regulates secretory traffic at the recycling endosomes and is involved in the infection of DV2 [3, 15]. Furthermore Myo5c, which like Myo5b belongs to the class V myosins, facilitates the exocytosis in lacrimal gland acinar cells [10]. Thus, these reports suggest that Myo5c may be involved in the intracellular transport and/or release of DV2. Here, we provide the experimental evidence on the involvement of Myo5c in the infection. Expression of the Myo5c tail could reduce the release, but not the production, of DV2 at 8, 24 and $48 \mathrm{~h}$ p.i. Since Myo5c is just defined as a low duty ratio, nonprocessive molecular motor [16], it is thought that the efficiency of the direct decrease of the viral release is relatively low, unlike the efficient Myo5b.

Furthermore, although the colocalization of Myo5c with DV2 antigen is rarely observed, Myo5c colocalizes with Rab8 and the functional inhibition of Myo5c leads to a significant increase of Rab8 in HepG2 cells. In combination with our previous finding that the interruption of the balance of Rab8 in host cells is able to inhibit the process of the DV2 infection [3], we think that Myo5c may utilize Rab8 to participate in the modulation of DV2 release in HepG2 cells though recycling endosomes or other Golgi-derived vesicular. However, further studies 
are needed to clarify the detailed mechanism during this process.

To our knowledge, this is the first report on the involvement of Myo5c in viral infection. The distribution of endogenous Myo5c is altered by DV2 in HepG2 cells and the viral release is reduced by the expression of the Myo5c mutant associated with the increase of Rab8. These observations may provide novel insight into the pathogenesis on intracellular traffic of DV and could be helpful in the development of antiviral drugs.

\section{Acknowledgments}

This work was supported by grants 30671853,30872227 and 30800983 from the National Natural Science Foundation of China, 5082004 from Beijing Natural Science Foundation, CSTC 2008BB5113 from the Natural Science Foundation of Chongqing and a funding project for academic human resources development in institutions of higher learning under the municipal jurisdiction of Beijing.

\section{References}

- Clyde K, Kyle JL, Harris E: Recent advances in deciphering viral and host determinants of dengue virus replication and pathogenesis. J Virol 2006;80:11418-11431.

2 Wang JL, Chen W, Wan YJ, Chen ZT, Zhang JL, Peng T, Gao N, An J: Dynamic changes in the monolayer permeability and rearrangement microfilaments in ECV304 cells after dengue virus infection (in Chinese). Chin J Zoonoses 2005;21:953-957.

-3 Xu XF, Chen ZT, Zhang JL, Chen W, Wang JL, Tian YP, Gao N, An J: Rab8, a vesicular traffic regulator, is involved in dengue virus infection in HepG2 cells. Intervirology 2008; 51:182-188.

4 Peränen J, Auvinen P, Virta H, Wepf R, Simons K: Rab8 promotes polarized membrane transport through reorganization of actin and microtubules in fibroblasts. J Cell Biol 1996;135:153-167.

$\checkmark 5$ Rodriguez OC, Cheney RE: Human myosin$\mathrm{Vc}$ is a novel class $\mathrm{V}$ myosin expressed in epithelial cells. J Cell Biol 2002;115:991-1004.

6 Kuo CH, Tai DI, Chang-Chien CS, Lan CK, Chiou SS, Liaw YF: Liver biochemical tests and dengue fever. Am J Trop Med Hyg 1992; 47:265-270.
7 Marianneau P, Cardona A, Edelman L, Deubel V, Despres P: Dengue virus replication in human hepatoma cells activates NF-кB which in turn induces apoptotic cell death. J Virol 1997;71:3244-3249.

-8 Marianneau P, Megret F, Olivier R, Morens DM, Deubel V: Dengue 1 virus binding to human hepatoma HepG2 and simian Vero cell surfaces differs. J Gen Virol 1996;77: 2547-2554.

9 Rosen L, Khin MM, U T: Recovery of virus from the liver of children with fatal dengue: reflections on the pathogenesis of the disease and its possible analogy with that of yellow fever. Res Virol 1989;140:351-360.

10 Marchelletta RR, Jacobs DT, Schechter JE, Cheney RE, Hamm-Alvarez SF: The class V myosin motor, myosin $5 \mathrm{c}$, localizes to mature secretory vesicles and facilitates exocytosis in lacrimal acini. Am J Physiol Cell Physiol 2008;295:13-28.

-11 Huber LA, Pimplikar S, Parton RG, Virta H, Zerial M, Simons K: Rab8, a small GTPase involved in vesicular traffic between the TGN and the basolateral plasma membrane. J Cell Biol 1993;123:35-45.
12 Hattula K, Furuhjelm J, Tikkanen J, Tanhuanpää K, Laakkonen P, Peränen J: Characterization of the Rab8-specific membrane traffic route linked to protrusion formation. J Cell Sci 2006;119:4866-4877.

13 Brock SC, Goldenring JR, Crowe JE Jr: Apical recycling systems regulate directional budding of respiratory syncytial virus from polarized epithelial cells. Proc Natl Acad Sci USA 2003;100:15143-15148.

14 Varthakavi V, Smith RM, Martin KL, Derdowski AL, Lapierre LA, Goldenring JR, Spearman P: The pericentriolar recycling endosome plays a key role in Vpu-mediated enhancement of HIV-1 particle release. Traffic 2006;7:298-307.

15 Henry L, Sheff DR: Rab8 regulates basolateral secretory, but not recycling, traffic at the recycling endosome. Mol Biol Cell 2008;19: 2059-2068.

16 Takagi Y, Yang Y, Fujiwara I, Jacobs D, Cheney RE, Sellers JR, Kovács M: Human myosin $\mathrm{Vc}$ is a low duty ratio, nonprocessive molecular motor. J Biol Chem 2008;283: 8527-8537. 